\title{
CDISC SDTM Pharmacogenomics/Genetics Findings Result Category Terminology
}

National Cancer Institute

\section{Source}

National Cancer Institute. CDISC SDTM Pharmacogenomics/Genetics Findings Result

Category Terminology. NCI Thesaurus. Code C135013.

Terminology associated with the pharmacogenomics/genetics findings result category codelist of the Clinical Data Interchange Standards Consortium (CDISC) Study Data Tabulation Model (SDT M). 